\title{
Multifrequency plane, nonlinear, and dissipative waves at arbitrary distances
}

Claes M. Hedberga)

Department of Mechanical Engineering, University of Karlskrona-Ronneby, 37179 Karlskrona, Sweden

(Received 6 November 1998; accepted for publication 16 August 1999)

\begin{abstract}
A solution for multifrequency plane waves propagating through a dissipative and nonlinear medium is presented. It originates from the well-known Bessel function series ratio for a pure sinusiodal wave, introduced by Cole and Mendousse. The solution is exact. The only limitation, inherited from the single-frequency solution, is the slow convergence of the series when the nonlinearity is very large compared to the dissipation. Otherwise any frequencies, amplitudes and phases can be introduced in the original wave and the solution is valid for any propagated distance. (C) 1999 Acoustical Society of America. [S0001-4966(99)02412-1]
\end{abstract}

PACS numbers: 43.25.Lj, 43.25.Cb [MFH]

\section{LIST OF SYMBOLS}

$A_{n}^{(l)} \quad=I_{n}\left(\alpha_{l} / 2 \alpha_{l} \epsilon^{*}\right)(-1)^{n} \exp \left(\right.$ in $\left.\gamma_{l}\right)$

a amplitude of single-frequency boundary condition

$a_{l} \quad$ amplitude of frequency $\alpha_{l}$ in boundary condition

$b \quad$ the effect of viscosity and heat conduction

$b_{l} \quad$ amplitude coefficient in boundary condition

$c_{0}$ the undisturbed sound velocity in the medium

$c_{k} \quad$ frequency-dependent coefficient in $U$ and $V$

$I_{n} \quad$ the modified Bessel function

$k \quad$ the frequency parameter in the sum (or integral) that is the solution $U$ summation index summation variable belonging to frequency $\alpha_{l}$ propagated distance

$=S(\sigma)$, the factor dependent on distance $\sigma$ in the separation of $U(\sigma, \theta)$

$T=T(\theta)$, the factor dependent on retarded time $\theta$ in the separation of $U(\sigma, \theta)$

$U$ transformation variable defined by $V=2 \epsilon \partial /$ $\partial \theta(\ln U)$

$U_{m f q}$ the subscript $m f q$ stresses that the solution is for multifrequency boundary condition, as opposed to solutions for single frequencies.

$V \quad=\mathrm{v} / \mathrm{v}_{0}$, dimensionless velocity amplitude

$V_{l} \quad$ the subscript $l$ is the solution for the frequency denoted by $l$ with frequency $\alpha_{l}$

\section{INTRODUCTION}

Nonlinear evolution of plane waves through a dissipative medium is described by the Burgers equation. For a single-frequency source solutions have been known for a long time. There are, as the best known examples, the Fubini solution ${ }^{1}$ valid for propagation up to shock formation and the Fay solution, ${ }^{2}$ whose validity is starting at approximately four shock distances. Both of these are Fourier series expres-

${ }^{a)}$ Electronic mail: claes.hedberg@ima.hk-r.se
$V_{m f q}$ the subscript $m f q$ stresses that the solution is for multifrequency boundary condition, as opposed to solutions for single frequencies

$V^{*} \quad=\mathrm{v} / \mathrm{v}_{00}$, a specific choice of dimensionless velocity amplitude

$\checkmark \quad$ velocity amplitude

$\mathrm{v}_{0} \quad$ characteristic velocity of the medium

$\alpha \quad$ integer angular frequency

$\alpha_{l} \quad$ integer angular frequency belonging to the frequency denoted by $l$ in the boundary condition

$\beta=(\gamma+1) / 2$, the nonlinearity of the medium

$\gamma \quad$ phase in boundary condition

$\gamma_{l} \quad$ phase in boundary condition belonging to frequency

$\alpha_{l}$

$\epsilon=b \omega / 2 \beta c_{0} \mathrm{v}_{0} \rho_{0}$, a dimensionless ratio between dissipation and nonlinearity

$\epsilon^{*} \quad=b \omega_{0} / 2 \beta c_{0} \mathrm{\vee}_{00} \rho_{0}$, a specific choice of $\epsilon$

$\theta \quad=\omega \tau$, dimensionless time

$\theta^{*} \quad=\omega_{0} \tau$, specific choice of dimensionless time

$\rho_{0} \quad$ undisturbed density of the medium

$\sigma \quad=\beta \omega \mathrm{V}_{0} x / c_{0}^{2}$, dimensionless propagation distance

$\sigma^{*} \quad=\beta \omega_{0} \vee_{00} x / c_{0}^{2}$, specific choice of dimensionless propagation distance

$\tau \quad=t-x / c_{0}$, retarded time

$\omega \quad$ characteristic angular frequency of the signal

$1 / \omega \quad$ a characteristic time

sions and neither one is exact. Both may be derived ${ }^{3}$ from the solution presented by Cole ${ }^{4}$ and Mendousse, ${ }^{5}$ which is a ratio between two Fourier series with Bessel function coefficients. This solution has one disadvantage in that the convergence of the series is poor ${ }^{6}$ and the solution does not work well when the nonlinearity is very large compared to the dissipation.

The propagation in a nonlinear and dissipative medium is a contest between the nonlinearity and the dissipation. Nonlinearity makes the positive amplitudes go faster than the equilibrium and the negative go slower. This results in the 
creation of combination frequencies of the original frequencies and their higher harmonics. Thus, most often also lower frequencies appear. The higher harmonics may turn the wave into a shocked state.

In contrast, dissipation is attenuating the higher harmonics much more than lower, thus making the waves go into shock less easily.

For original signals consisting of several frequencies there has been little more than numerics to use with a few exceptions for the case of no dissipation. There is one originating from Burgers for zero dissipation, ${ }^{7}$ where the total wave solution can be obtained for all distances through the finding of the maximum of a function. The most distinguished result is the analytic solution by Fenlon ${ }^{8}$ for zero dissipation, valid up to the formation of shock, which is a generalization of the Fubini solution. The solution to the Burgers equation in the form of a ratio between two infinite integrals yields a complete solution for the given distance. The integration interval naturally has to be much smaller and the integrand ought to be examined beforehand in order to reach accurate results. In this paper a solution for multifrequency conditions for dissipative and nonlinear propagation is introduced. It originates from the single-frequency Cole-Mendousse solution and it is a generalization of the same. Therefore the derivation of the multi-frequency solution begins with the well-known derivation of the singlefrequency solution.

\section{THE SOLUTION FOR ONE FREQUENCY}

The nonlinear equation for plane waves in a homogeneous dissipative medium is Burgers' equation, given in dimensionless variables as

$$
\frac{\partial V}{\partial \sigma}-V \frac{\partial V}{\partial \theta}-\epsilon \frac{\partial^{2} V}{\partial \theta^{2}}=0 .
$$

The definition of the dimensionless variables - using a characteristic velocity $\mathrm{v}_{0}$ of the medium, $b$ the effect of viscosity and heat conduction, $\tau=t-x / c_{0}$ the retarded time, $\rho_{0}$ the undisturbed density, $c_{0}$ the undisturbed sound velocity, $\beta$ $=(\gamma+1) / 2$ the nonlinearity for a fluid and a characteristic time $1 / \omega-$ are

$$
\begin{aligned}
V & =\frac{\mathrm{v}}{\mathrm{v}_{0}}, \\
\theta & =\omega \tau, \\
\sigma & =\frac{\beta}{c_{0}^{2}} \omega \mathrm{v}_{0} x, \\
\epsilon & =\frac{1}{2 \beta} \frac{b \omega}{c_{0} \mathrm{v}_{0} \rho_{0}} .
\end{aligned}
$$

If the dimensionless ratio between dissipation and nonlinearity $\epsilon$ is less than 1 , nonlinear theory should be used. Equation (1) can be transformed into a linear equation through

$$
V=2 \epsilon \frac{\partial U / \partial \theta}{U}=2 \epsilon \frac{\partial}{\partial \theta}(\ln U),
$$

and the heat conduction equation is obtained,

$$
\frac{\partial U}{\partial \sigma}=\epsilon \frac{\partial^{2} U}{\partial \theta^{2}} .
$$

This transformation was found by Hopf ${ }^{9}$ and Cole. ${ }^{4}$

The solution can be found by separation of the variables:

$$
U(\sigma, \theta)=S(\sigma) T(\theta) .
$$

The general solution of (7) can then be written as a sum,

$$
U=\sum_{k=-\infty}^{\infty} c_{k} e^{-\epsilon k^{2} \sigma} e^{i k \theta}
$$

or as an integral,

$$
U(\sigma, \theta)=\int_{-\infty}^{\infty} c(k) e^{i k \theta-\epsilon k^{2} \sigma} d k .
$$

Before turning to the Fourier series form a short discussion on the integral form will be conducted. It is a general solution, but in practice there is numerical trouble encountered when solving this integral because the exponent in the infinite integral will vary greatly. As an example, let the boundary condition be a simple sine wave:

$$
V(\sigma=0, \theta)=\sin \theta .
$$

This results through (6) and (1) ${ }^{10}$ in the solution

$$
V=2 \frac{\int_{-\infty}^{\infty} \frac{\theta^{\prime}-\theta}{\sigma} \exp \left[\frac{1}{2 \epsilon}\left(-\cos \theta^{\prime}\right)-\frac{\left(\theta-\theta^{\prime}\right)^{2}}{4 \epsilon \sigma}\right] d \theta^{\prime}}{\int_{-\infty}^{\infty} \exp \left[\frac{1}{2 \epsilon}\left(-\cos \theta^{\prime}\right)-\frac{\left(\theta-\theta^{\prime}\right)^{2}}{4 \epsilon \sigma}\right] d \theta^{\prime}} .
$$

This is a simple solution to Burgers' equation. The limits in the integration has to be decreased considerably as the numbers may become very large, in particular, for small $\epsilon$. The way to proceed is to examine the exponents beforehand to be able to choose the relevant interval for the integration. The integration interval may often have to be divided into several smaller intervals. This procedure leads naturally to the saddle point method concept which only takes into account the major parts of the integrand around certain maxima. Through the saddle point method both the Fubini and Fay solutions may be derived. The Fubini through use of one saddle point ${ }^{11}$ and the Fay through two saddle points yielding the time domain Khokhlov solution, ${ }^{12}$ which is then converted into a Fourier series. ${ }^{10}$ For the simple sine wave there is only one maximum per period. For more complicated input the analytical saddle point method leads to solutions valid only locally, ${ }^{13}$ except for nondissipative continuous waves before the formation of shocks.

The Fourier series representation (9) is appropriate when the boundary condition is periodical. For the simple harmonic signal as a boundary condition (11) the solution to Eq. (1) was obtained by Cole ${ }^{4}$ and Mendousse: ${ }^{5}$ 


$$
V(\sigma, \theta)=-4 \epsilon \frac{\sum_{n=1}^{\infty} \exp \left(-n^{2} \epsilon \sigma\right) n(-1)^{n} I_{n}(1 / 2 \epsilon) \sin n \theta}{I_{0}(1 / 2 \epsilon)+2 \sum_{n=1}^{\infty} \exp \left(-n^{2} \epsilon \sigma\right)(-1)^{n} I_{n}(1 / 2 \epsilon) \cos n \theta} .
$$

Here, $I_{n}$ denotes modified Bessel functions.

Of special interest might be to replace the quotient between two Fourier series (13) by an analytic single Fourier series. This has been done by Enflo $^{3}$ through a recursion algorithm

\section{SOLUTION FOR SEVERAL FREQUENCIES}

Any solution for a periodic boundary condition may have the form of the ansatz (9):

$$
U_{m f q}=\sum_{k=-\infty}^{\infty} c_{k} e^{-k^{2} \epsilon \sigma} e^{i k \theta}
$$

where the subscript $m f q$ stands for multifrequency. This is the equivalent to the solution

$$
V_{m f q}(\sigma, \theta)=i 2 \epsilon \frac{\sum_{k=-\infty}^{\infty} k c_{k} e^{-k^{2} \epsilon \sigma} e^{i k \theta}}{\sum_{k=-\infty}^{\infty} c_{k} e^{-k^{2} \epsilon \sigma} e^{i k \theta}} .
$$

Let (15) be the solution to a boundary condition consisting of a number of $L$ frequencies, the $\alpha_{l}$ 's are integers:

$$
V_{m f q}(\sigma=0, \theta)=\sum_{l=-L}^{L} b_{l} e^{i \alpha_{l} \theta} .
$$

Each one of the $L$ frequencies has on their own a known solution $V_{l}$ in a form similar to (13).

Because this is a nonlinear problem they cannot be superposed to form the solution to the multiple frequency boundary condition-except at the distance $\sigma=0$. At this distance no nonlinear (nor linear) evolution has taken place, and superposition is allowed. So this is where, in the remainder of this section, the coefficients of the multifrequency wave will be extracted from the known coefficients of the single frequencies.

Some care must be taken to make sure the individual $V$ 's are expressed in the same dimensionless variables because, if the dimensionless variables of each individual frequency are inserted straight into (13), these dimensionless variables will be defined for each particular frequency. They will then not stand for the same real variables. If they are to be combined they must be expressed by some definite variables-the same for all individual frequencies. Let the choices be [compare (2)-(5)]

$$
\begin{aligned}
V^{*} & =\frac{\mathrm{v}}{\mathrm{v}_{00}}, \\
\theta^{*} & =\omega_{0} \tau, \\
\sigma^{*} & =\frac{\beta}{c_{0}^{2}} \omega_{0} \mathrm{v}_{00} x, \\
\epsilon^{*} & =\frac{1}{2 \beta} \frac{b \omega_{0}}{c_{0} \mathrm{v}_{00} \rho_{0}} .
\end{aligned}
$$

All single-frequency boundary conditions written like

$V^{*}\left(\sigma^{*}=0, \theta^{*}\right)=\alpha \sin \left(\alpha \theta^{*}+\gamma\right)$

$$
=\frac{a}{2}\left[\exp \left(i\left(\alpha \theta^{*}+\gamma\right)\right)-\exp \left(-i\left(\alpha \theta^{*}+\gamma\right)\right)\right],
$$

will get the results in the same dimensionless parameters by making the following replacements in (13),

$$
\begin{aligned}
V & =\frac{V_{*}}{a}, \\
\theta & =\alpha \theta^{*}+\gamma, \\
\sigma & =a \alpha \sigma^{*}, \\
\epsilon & =\frac{\alpha}{a} \epsilon^{*} .
\end{aligned}
$$

So the solutions to the individual frequencies will be

$$
V^{*}=a V\left(\sigma^{*}, \theta^{*}\right)=-4 \alpha \epsilon^{*} \frac{\sum_{n=1}^{\infty} \exp \left(-n^{2} \alpha^{2} \epsilon^{*} \sigma^{*}\right) n(-1)^{n} I_{n}\left(\frac{a}{2 \alpha \epsilon^{*}}\right) \sin n\left(\alpha \theta^{*}+\gamma\right)}{I_{0}\left(\frac{a}{2 \alpha \epsilon^{*}}\right)+2 \sum_{n=1}^{\infty} \exp \left(-n^{2} \alpha^{2} \epsilon^{*} \sigma^{*}\right)(-1)^{n} I_{n}\left(\frac{a}{2 \alpha \epsilon^{*}}\right) \cos n\left(\alpha \theta^{*}+\gamma\right)} .
$$

Now we may write the superposition at zero propagated distance (15),

$$
\begin{aligned}
V_{m f q}^{*}\left(\sigma^{*}=0, \theta^{*}\right) & =i 2 \epsilon^{*} \frac{\sum_{k=-\infty}^{\infty} k c_{k} e^{i k \theta^{*}}}{\sum_{k=-\infty}^{\infty} c_{k} e^{i k \theta^{*}}}, \\
& =\sum_{l=1}^{L} V_{l}^{*}\left(\sigma^{*}=0, \theta^{*}\right)=\{\text { with }(26)\},
\end{aligned}
$$




$$
\begin{aligned}
& =\sum_{l=1}^{L} \frac{\sum_{n=1}^{\infty}-2 \alpha_{l} \epsilon^{*} n(-1)^{n} I_{n}\left(\frac{a_{l}}{2 \alpha_{l} \epsilon^{*}}\right)\left[\exp \left(\operatorname{in}\left(\alpha_{l} \theta^{*}+\gamma_{l}\right)\right)-\exp \left(-i n\left(\alpha_{l} \theta^{*}+\gamma_{l}\right)\right)\right]}{\sum_{n=1}^{\infty}(-1)^{n_{l}} I_{n}\left(\frac{a_{l}}{2 \alpha_{l} \epsilon^{*}}\right)\left[\exp \left(\operatorname{in}\left(\alpha_{l} \theta^{*}+\gamma_{l}\right)\right)+\exp \left(-i n\left(\alpha_{l} \theta^{*}+\gamma_{l}\right)\right)\right]}, \\
& =\sum_{l=1}^{L}-2 \alpha_{l} \epsilon^{*} \frac{\sum_{n=-\infty}^{\infty} n(-1)^{n} I_{n}\left(\frac{a_{l}}{2 \alpha_{l} \epsilon^{*}}\right) \exp \left(i n\left(\alpha_{l} \theta^{*}+\gamma_{l}\right)\right)}{\sum_{n=-\infty}^{\infty}(-1)^{n} I_{n}\left(\frac{a_{l}}{2 \alpha_{l} \epsilon^{*}}\right) \exp \left(\operatorname{in}\left(\alpha_{l} \theta^{*}+\gamma_{l}\right)\right)}, \\
& =\sum_{l=1}^{L}-2 \alpha_{l} \epsilon^{*} \frac{\sum_{n=-\infty}^{\infty} n A_{n}^{(l)} \exp \left(\text { in } \alpha_{l} \theta^{*}\right)}{\sum_{n=-\infty}^{\infty} A_{n}^{(l)} \exp \left(\operatorname{in} \alpha_{l} \theta^{*}\right)},
\end{aligned}
$$

with

$$
A_{n}^{(l)}=I_{n}\left(\frac{a_{l}}{2 \alpha_{l} \epsilon^{*}}\right)(-1)^{n} \exp \left(\text { in } \gamma_{l}\right) .
$$

The relation between $A_{n}$ and $A_{-n}$ is

$$
A_{-n}^{(l)}=A_{n}^{(l)} \exp \left(-i 2 n \gamma_{l}\right) .
$$

The next step is to identify (27) with (31) in each frequency, with $n^{(l)}$ being the $n$ belonging to frequency $\alpha_{l}$, and $A_{n}^{(l)}$,

$$
k=n^{(1)} \alpha_{l}+n^{(2)} \alpha_{2}+\cdots+n^{(L)} \alpha_{L}=\sum_{l=1}^{L} n^{(l)} \alpha_{l},
$$

As the numerator is simply the derivative of the denominator, the identification may be done in either the numerator or denominator, yielding

$$
\begin{aligned}
\sum_{k=-\infty}^{\infty} c_{k} e^{i k \theta^{*}}= & \sum_{n^{(1)}=-\infty}^{\infty} A_{n}^{(1)} \exp \left(i n^{(1)} \alpha_{1} \theta^{*}\right) \\
& \times \sum_{n^{(2)}=-\infty}^{\infty} A_{n}^{(2)} \exp \left(i n^{(2)} \alpha_{2} \theta^{*}\right) \cdots \\
& \sum_{n^{(L)}=-\infty}^{\infty} A_{n}^{(L)} \exp \left(i n^{(L)} \alpha_{L} \theta^{*}\right) .
\end{aligned}
$$

This equation is solved for each $k$. The coefficients become

$$
c_{k}=\sum_{k=\sum n^{(l)} \alpha_{l}} A_{n}^{(1)} A_{n}^{(2)} \cdots A_{n}^{(L)} .
$$

Equation (36), together with (15), is the solution to Burgers' equation (1), valid for any number of frequencies with arbitrary amplitudes and phases.

This new solution has many advantages. It is the first exact multifrequency solution in explicit form, which includes dissipation. It is suitable as a replacement for numerical methods. It works fast on a computer, from a couple of seconds for two initial frequencies up to a few minutes for 200. Once the solution is obtained, it is valid for all distances (which, of course, is difficult to match by pure numerics).

Approximate analytical expressions describing the behavior in limiting cases might, of course, be derived from this exact solution. But as the parameters in a multifrequency condition contains many parameters like relative amplitudes, relative frequencies, and relative phases, one cannot expect to arrive at general asymptotic expressions. Actually, the exact individual frequencies in form of a straightforward Fourier series may be obtained analytically in the same way as for a single-frequency. ${ }^{3}$ But the final analytical results would be intricate and is therefore not included in this article.

The most analytical way of calculating the coefficients $A_{n}^{(l)}$ is through the modified Bessel functions in the explicit solution (13). A faster way on a computer is to use a recursion method, formulated by Gallia, which is described in Appendix A.

When the dissipation $\epsilon^{*}$ becomes small, a larger number of terms will have to be included in the series. Due to the slow convergence of these terms and the difference in size of the coefficients, there is a lower limit in $\epsilon$ below which the calculations lead to numbers exceeding the numerical representation in computers. This is the only limitation in the presented method, the same as for the Cole-Mendousse solution (13).

\section{NUMERICAL RESULTS}

In this section we present some results showing the evolution of multifrequency signals. Comparisons with numerical and analytical methods are made. The numerical method's accuracy is validated in Appendix B, where it is compared to well-known single-frequency solutions.

The first example is $V_{0}=\sin 7 \theta+\sin 9 \theta$ for the dissipation over nonlinearity ratio $\epsilon^{*}=0.05$, where the difference frequency, $9-7=2$, is not the same as the lowest frequency, which is equal to 1 .

In Fig. 1 it is seen how combination frequencies are created. Note how the lowest frequency is not noticable, as it cannot be created directly from 7 and 9 . It is created from, e.g., $(4 \times 7=28)-(3 \times 9=27)=1$, giving a very small contribution. After some distance, $\sigma=5$, the difference fre- 


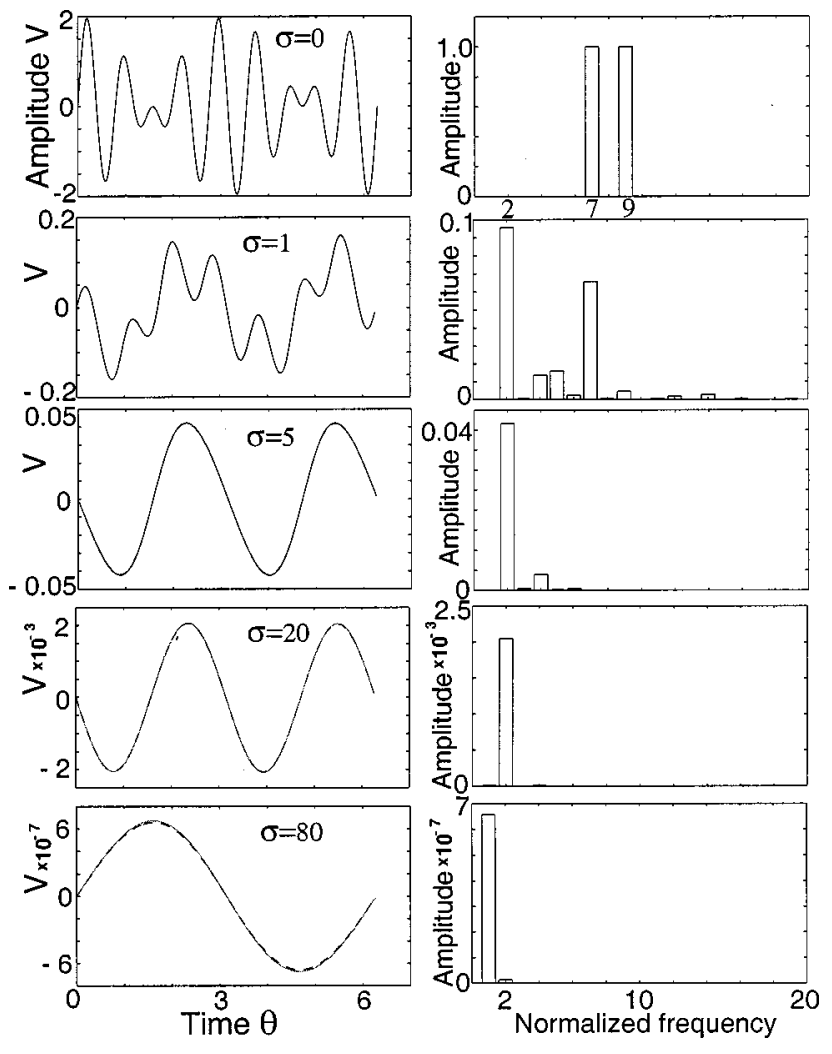

FIG. 1. The difference frequency $9-7=2$ is not the same as the lowest frequency equal to 1 . Here $V_{0}=\sin 7 \theta+\sin 9 \theta, \epsilon^{*}=0.05$. The numerical solution is a dashed line and the multi frequency solution is solid.

quency has become dominant and many of the higher harmomics have been damped out. The wave will stay in this approximate shape for a long distance; see, for example, $\sigma$ $=20$. One might believe this to be the final stage. But later on, at $\sigma=80$, the higher damping of frequency 2 compared to that of frequency 1 , has made the lowest frequency the only one remaining. The comparison with a numerical algorithm indicates that both solutions seem to be correct.

To make a comparison with the Fenlon solution, it is necessary to stay in the preshock region, which in this case means up to the distance $\sigma_{s} \approx 1 /(7+9)=0.0625$. In Fig. 2 is the multifrequency solution and the numerical solution for a dissipation of $\epsilon=0.05$ and the Fenlon solution, which is inherently for zero dissipation, is shown. The expected difference is present.

Next, the input is an $N$-wave that is represented in the initial wave with 210 frequencies; see Fig. 3. The calculation is also performed with 210 frequencies and is compared with

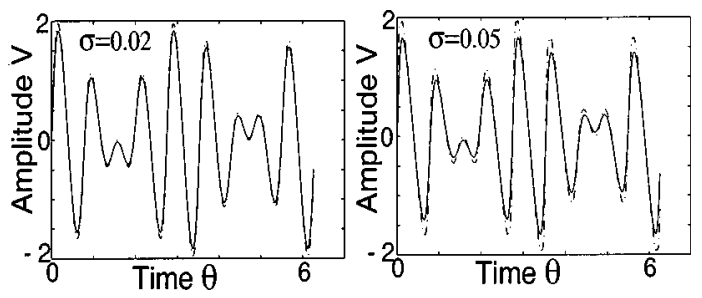

FIG. 2. The present solution (solid), the numerical solution (dashed), and the Fenlon solution (dash-dotted) in its region of validity before shock formation, $V_{0}=\sin 7 \theta+\sin 9 \theta, \epsilon^{*}=0.05$.

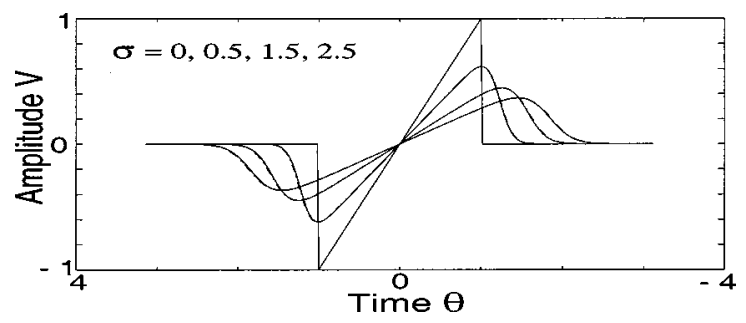

FIG. 3. The initial $N$ wave and its evolution according to the multifrequency solution (solid), compared with a numerical algorithm (dash-dotted), $\epsilon^{*}$ $=0.04$.

a numerical solution. This shows not only that a large number of harmonics may be used in the input but also that the propagation of pulses are possible to calculate.

\section{CONCLUSIONS}

A solution for multifrequency plane waves propagating through a dissipative and nonlinear medium has been presented. Any frequencies, number of frequencies, amplitudes, and phases can be chosen as input. It originates from a solution for a pure sinusiodal wave, known from Cole and Mendousse, expressed as a ratio between Bessel function series. The solution for multiple frequencies is calculated from the knowledge that the solution has to have a specific form and that the unknown coefficients in this form can be extracted at zero distance from the sum of the single-frequency solutions. As the single frequency solutions are exact, so is the solution for the multiple-frequency waves. For the same reason, the limitation in that the series converge slowly when the dissipation over nonlinearity ratio is very large is inherited from the single-frequency solution. The solution depends on the separation of variables, which means that once the coefficients are calculated, one automatically has a solution valid for any distance.

The validity of the method has been shown by comparison to a numerical algorithm. The method is considerably faster than regular numerical methods for large distances, in particular, when the number of input frequencies are not great.

The solution lends itself to the extraction of analytical limiting solutions for any given set of parameters.

\section{ACKNOWLEDGMENTS}

I was inspired to do this work when looking at the recursion formula work by Ken Gallia, University of Texas at Austin. The idea came to me after my postdoc years 19951997 at the University of Texas supported by TFR (the Swedish Research Council for Engineering Sciences), but the work there prepared me for this article. I would like to thank the people at the Acoustics group at the University of Texas and especially my mentor Bengt Enflo, KTH, for his continual advice.

\section{APPENDIX A: THE BESSEL RECURSION ALGORITHM}

This appendix describes the recursion formula developed by Gallia at the University of Texas. 
Put Eq. (21) equal to a solution of the form (15) at $\sigma$ $=0$ :

$$
\begin{aligned}
V(\sigma=0, \theta)=\sin \theta & =\frac{1}{i 2} e^{i n \theta}-\frac{1}{i 2} e^{i n \theta} \\
& =i 2 \epsilon \frac{\sum_{k=-\infty}^{\infty} k A_{k} e^{i k \theta}}{\sum_{k=-\infty}^{\infty} A_{k} e^{i k \theta} .}
\end{aligned}
$$

Multiply by the denominator

$$
i 2 \epsilon \sum_{k=-\infty}^{\infty} k A_{k} e^{i k \theta}=\sum_{m=-\infty}^{\infty} A_{m} e^{i m \theta}\left[\frac{1}{i 2} e^{i n \theta}-\frac{1}{i 2} e^{-i n \theta}\right] .
$$

Here the coefficients $A_{k}$ are regarded as unknowns. One of the possible methods of obtaining these coefficients is to solve this as a system of equations.

But the fastest way to get the solution is through the identification of the exponents giving us the recursion formula

$$
-4 \epsilon k A_{k}=A_{k-1}-A_{k+1},
$$

or, after rearrangement and insertion of the chosen normalized variable $\epsilon^{*}$ from (20),

$$
A_{k-1}=A_{k+1}+4 \frac{\alpha \epsilon^{*}}{a} k A_{k} .
$$

Starting with almost any values of $A_{K+K \text { plus }}$ and $A_{K+K \text { plus }-1}$, where $K$ is the number of terms in the series and $K_{\text {plus }}$ is approximately 10, the coefficients are calculated backward. The recursion ends with

$$
A_{0}=A_{2}+4 \frac{\alpha \epsilon^{*}}{a} A_{1} \text {. }
$$

These coefficients have been multiplied with a factor that is dependent on the starting values. But as the final solution is a ratio this factor will disappear.

\section{APPENDIX B: EVALUATION OF NUMERICAL METHOD}

The numerical program used to validate the multifrequency solution is a split step superposition algorithm in which the nonlinear part is performed in time domain and the dissipative in frequency domain. The nonlinear part is an exact solution to Eq. (1) with the third term absent and the dissipative part is the solution to (1) when the second term is absent. The numerical code will, in this appendix itself, be validated by comparisons with known solutions existing for a single frequency. Three solutions are represented in Fig. B1. At all distances is present the Cole-Mendousse solution and the numerical solution. At distance $\sigma=0.5$ the third solution is the Fubini solution. The Fubibi solution does not include dissipation, which makes it differ from the other two
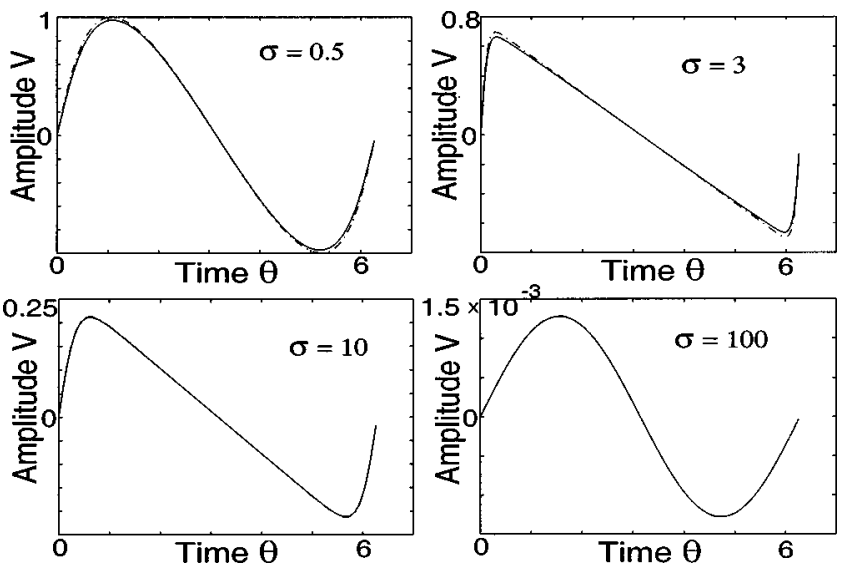

FIG. B1. Here $V_{0}=\sin \theta, \epsilon^{*}=0.05$. The Cole-Mendousse solution (solid line), the numerical solution (dashed), and, at $\sigma=0.5$, the Fubini solution (dash-dotted); at $\sigma=3,10$, and 100 the Fay solution (dash-dotted). The solutions coincides well at the distances where they are valid.

primarily by its larger amplitude. At distances $\sigma=3,10$, and 100 the third solution is the Fay solution. For the distance $\sigma=3$ the Fay solution is still not very close to being exact, as its lower limit of validity is approximately $\sigma=4$, which means that at $\sigma=10$ all three solutions coincide. The Fay and the Cole-Mendousse solutions are valid for indefinitely large values. At $\sigma=100$, the numerical solution is coinciding fairly well, which proves its stability and suitability as a comparing tool for the multifrequency solution.

${ }^{1}$ G. E. Fubini, "Anomalie nella propagazione di onde acustiche di grande ampiezza," Alta Freq. 4, 530 (1935).

${ }^{2}$ R. D. Fay, "Plane sound waves of finite-amplitude," J. Acoust. Soc. Am. 3, 222 (1931).

${ }^{3}$ B. O. Enflo, "Some analytical results on nonlinear acoustic wave propagation in diffusive media," Radiofizika 36, 665-686 (1993).

${ }^{4} \mathrm{~J}$. D. Cole, "'On a quasi-linear parabolic equation occurring in aerodynamics,’ Q. Appl. Math. 9, 225-236 (1951).

${ }^{5}$ J. S. Mendousse, "Nonlinear dissipative distortion of progressive sound waves at moderate amplitudes," J. Acoust. Soc. Am. 25, 51-54 (1953).

${ }^{6} \mathrm{D}$. T. Blackstock, "Thermoviscous attenuation of plane, periodic, finiteamplitude sound waves,' J. Acoust. Soc. Am. 36, 534-542 (1964).

${ }^{7}$ J. M. Burgers, The Nonlinear Diffusion Equation (Reidel, Dordrecht, 1977).

${ }^{8}$ F. H. Fenlon, "An extension of the Fubini series for a multiple-frequency CW acoustic source of finite amplitude," J. Acoust. Soc. Am. 51, 284289 (1972).

${ }^{9}$ E. Hopf, "The partial differential equation $u_{t}+u u_{x}=\mu$," Commun. Pure Appl. Math. 3, 201-230 (1950).

${ }^{10}$ O. V. Rudenko and S. I. Soluyan, Theoretical Foundations of Nonlinear Acoustics (Plenum, New York, 1977).

${ }^{11}$ C. M. Hedberg, '“One-saddle point solution to Burgers' equation for harmonic signals," submitted to J. Acoust. Soc. Am.

${ }^{12}$ S. I. Soluyan and R. V. Khokhlov, "Propagation of acoustic waves of finite amplitude in a dissipative medium, Vestn Mosk. Univ. (Series III)," Fiz. Astron. 3, 52-61 (1961); or in Beyer, Nonlinear Acoustics in Fluids (Van Nostrand Reinhold, New York, 1984), pp. 193-206.

${ }^{13}$ C. M. Hedberg, "Nonlinear propagation through a fluid of waves originating from a biharmonic sound source,' J. Acoust. Soc. Am. 96, 18211828 (1994) 